\title{
Razonamiento Científico y Conocimientos Conceptuales de Mecánica: Un Diagnóstico de Alumnos de Primer Ingreso a Licenciaturas en Ingeniería
}

\author{
María D. Rodríguez, Daniel A. Mena, Carlos M. Rubio \\ Facultad de Ingeniería Química, Universidad Autónoma de Yucatán, Periférico Nte. \\ Km. 33.5, Tablaje Catastral, 13615, Col. Chuburná de Hidalgo inn, C.P. 97203, \\ Mérida, Yucatán-México (e-mail: mmartin@uady.mx)
}

Recibido Sep. 14, 2010; Aceptado Oct. 21, 2010; Versión final recibida Nov. 24, 2010

\begin{abstract}
Resumen
Se presenta un diagnóstico del grado de razonamiento científico de cinco grupos de estudiantes de primer ingreso a licenciaturas en ingeniería aplicando la prueba de aula de razonamiento científico de Lawson. Se aplicó el Cuestionario sobre Conceptos de Fuerza (Force Concept Inventory) para diagnosticar su nivel de conocimiento conceptual en Mecánica. La aplicación del Cuestionario mostró porcentajes de aciertos bajos en todos los rubros, principalmente los relacionados con Segunda Ley de Newton. Además, se analizaron los resultados del examen de selección EXANI-II aplicado por el Centro Nacional de Evaluación para la Educación Superior de México. En este caso, las puntuaciones del índice de razonamiento lógico matemático y las puntuaciones del examen diagnóstico de física fueron también bajas.
\end{abstract}

\section{Scientific Reasoning and Conceptual Knowledge in Mechanics: A Diagnosis of Freshmen to Undergraduate Engineering}

\begin{abstract}
This work presents a diagnosis of the extent of scientific reasoning of five groups of freshmen in engineering, through the application of Lawson's classroom test of scientific reasoning. The Force Concept Inventory was applied to assess their level of conceptual knowledge in mechanics. The application of Force Concept Inventory showed low percentages of correct answers in all subjects, especially those related to Newton's Second Law. Also, the scores of the selection test EXANI-II implemented by the National Center for Higher Education Assessment in México were analyzed. In this case, the scores obtained in the mathematical logic reasoning index and the scores of the physics test were also low.
\end{abstract}

Keywords: Scientific Reasoning, conceptual knowledge, Mechanical, Diagnosis 


\section{INTRODUCCIÓN}

La resolución de problemas en física requiere tanto del conocimiento adecuado como de algún tipo de razonamiento (Concari, 2002). Tanto la comprensión profunda como la resolución de problemas eficiente surgen de ser capaces de analizar situaciones conceptualmente. Así, el análisis se vuelve el puente que permite que los conceptos sean útiles para la resolución de problemas. Una consecuencia de esta perspectiva es que los estudiantes pueden resolver problemas más difíciles que otros estudiantes que siguen el enfoque tradicional, y generalmente mejoran sus habilidades de razonamiento y desarrollan una comprensión más profunda de los conceptos y principios (Leonard, Gerace y Dufresne, 2002). Uno de los instrumentos más utilizados para medir el aprendizaje de conceptos en mecánica clásica introductoria es el inventario sobre el concepto de fuerza FCl (Hestenes, Wells, y Swackhamer, 1992). Esta prueba consta de 30 preguntas de selección múltiple que evalúan el concepto de fuerza a través de cuatro dimensiones: cinemática, primera, segunda y tercera Leyes de Newton. La tabla 1 muestra la clasificación de conceptos de mecánica newtoniana evaluados por el $\mathrm{FCl}$.

Hake utilizó el $\mathrm{FCl}$ en una gran población de estudiantes de nivel preuniversitario y universitario en Estados Unidos para medir el aprendizaje en cursos similares de mecánica introductoria (Hake, 1998). La aplicación del instrumento se hizo al inicio y al final del semestre con el propósito de cuantificar el aprendizaje conceptual a través de la ganancia normalizada

$$
g=\frac{\text { FCIpost }- \text { FCIpre }}{100-\text { FCIpre }}
$$

Este factor puede tomar valores entre 0 y 1 , donde 0 representa que no hay aprendizaje, mientras que 1 corresponde al máximo aprendizaje posible. La normalización que presenta este factor permite comparar el desempeño de cursos con poblaciones diferentes, como es el caso de cursos de mecánica en diferentes universidades, e incluso diferentes niveles educativos.

Por otro lado, en un trabajo clásico de educación en la física, Renner y Lawson hacen una revisión de la teoría Piaget que puede ayudar a entender cómo el razonamiento científico se relaciona con la instrucción (Renner y Lawson, 1973). De acuerdo a los estudios de Piaget, se observan cuatro estados de pensamiento que se van alcanzando progresivamente desde el nacimiento hasta que se alcanza la edad adulta. Cerca de los siete años de edad, el niño entra en el tercer estado de razonamiento Ilamado operacional concreto. En este estado el niño puede llevar a cabo experimentos mentales. Las operaciones que puede realizar son concretas en el sentido de que se relacionan directamente a objetos y no aún con hipótesis verbalizadas. El cuarto y último estado de Piaget se conoce como operacional formal y ocurre aproximadamente entre los 11 y 15 años. En este estado el individuo es capaz de razonar con proposiciones sin la necesidad de objetos. Para desarrollar este estado de pensamiento es necesario haber desarrollado previamente el pensamiento concreto. Un pensador formal puede formular hipótesis y probarlas. Para lograr esto debe aislar y controlar variables, así como excluir las variables irrelevantes.

Aunque en la actualidad, algunos autores consideran superado el enfoque de aprendizaje puramente piagetiano (Pozo, 2006) planteando distintos niveles de análisis del aprendizaje, desde la conducta, la información, la representación y el conocimiento bajo el punto de vista de las diferentes teorías psicológicas, el instrumento diseñado por Renner y Lawson sigue sido usado en trabajos relacionados con ciencias, especialmente física y matemáticas pues es una prueba que permite medir específicamente los niveles de razonamiento científico, siendo éste de suma importancia para la comprensión y avance en estas áreas (Diff y Tache, 2007, Coletta y Phillips, 2007). Renner y Lawson plantean que las abstracciones en física son modelos creados por científicos para explicar datos de mediciones (Renner y Lawson, 1973). Estos modelos no aparecen directamente de observaciones, sino que son intentos para construir una explicación o modelo del fenómeno observado. 
Tabla 1. Conceptos Newtonianos en FORCE CONCEPT INVENTORY (FCI)

\begin{tabular}{|c|c|c|}
\hline \multirow{5}{*}{ 0. Cinemática } & \multicolumn{2}{|c|}{ Velocidad discriminada de la posición } \\
\hline & \multicolumn{2}{|c|}{ Aceleración discriminada de la velocidad } \\
\hline & \multicolumn{2}{|c|}{ Aceleración constante implica órbita parabólica } \\
\hline & \multicolumn{2}{|c|}{ Cambio de velocidad } \\
\hline & \multicolumn{2}{|c|}{ Adición vectorial de velocidades } \\
\hline \multirow{4}{*}{$\begin{array}{l}\text { 1. Primera Ley de } \\
\text { Newton }\end{array}$} & \multicolumn{2}{|c|}{ Sin fuerzas } \\
\hline & \multicolumn{2}{|c|}{ Dirección constante de la velocidad } \\
\hline & \multicolumn{2}{|c|}{ Velocidad constante } \\
\hline & \multicolumn{2}{|c|}{ Con fuerzas que se cancelan } \\
\hline \multirow{2}{*}{$\begin{array}{l}\text { 2. Segunda ley de } \\
\text { Newton }\end{array}$} & \multicolumn{2}{|c|}{ Fuerza impulsiva } \\
\hline & \multicolumn{2}{|c|}{ Fuerza constante implica aceleración constante } \\
\hline \multirow{2}{*}{$\begin{array}{l}\text { 3. Tercera ley de } \\
\text { Newton }\end{array}$} & \multicolumn{2}{|c|}{ Para fuerza impulsivas } \\
\hline & \multicolumn{2}{|c|}{ Para fuerzas continuas } \\
\hline \multirow{2}{*}{$\begin{array}{l}\text { 4. Principio de } \\
\text { superposición }\end{array}$} & \multicolumn{2}{|c|}{ Suma de vectores } \\
\hline & \multicolumn{2}{|c|}{ Fuerzas que se cancelan } \\
\hline \multirow{7}{*}{ 5. Tipos de fuerza } & \multirow{3}{*}{$\begin{array}{l}\text { De contacto } \\
\text { entre sólidos }\end{array}$} & Pasivo \\
\hline & & Impulsivo \\
\hline & & Fricción opuesta al movimiento \\
\hline & \multirow{2}{*}{$\begin{array}{l}\text { De contacto } \\
\text { con fluidos }\end{array}$} & Resistencia del aire \\
\hline & & Presión del aire \\
\hline & \multirow{2}{*}{ Gravitación } & Aceleración independiente del peso \\
\hline & & Trayectoria parabólica \\
\hline
\end{tabular}

Un pensador formal podrá manejar estas abstracciones, pero no un pensador concreto. Con el propósito de medir el estado de pensamiento del estudiante diseñaron un instrumento con dos tipos de tareas. La primera es sobre la conservación del volumen, que da evidencia de un pensamiento formal de un principiante. La segunda tarea es sobre la exclusión de variables irrelevantes, que permite observar el uso de lógica proposicional, que es un prerrequisito para el entendimiento de abstracciones. Llevaron a cabo un experimento con estudiantes, de niveles preuniversitario y universitario, a quienes se les aplicó el instrumento, evidenciando las diferencias en el razonamiento científico de los estudiantes de diferentes niveles educativos. Esta prueba consta de 12 reactivos y ubica a los estudiantes en tres niveles de razonamiento: pensamiento empírico- inductivo ó concreto ( 0 a 4 aciertos), pensamiento en transición o formal ( 5 a 8 aciertos) y pensamiento hipotético-deductivo ó postformal (9 a 12 aciertos).

En esencia, esta prueba evalúa seis aspectos del razonamiento: conservación de magnitudes física, pensamiento de proporcionalidad, identificación y control de variables, pensamiento probabilístico, pensamiento combinatorio y pensamiento correlacional. Estos aspectos se evalúan de manera aislada ó combinada en las doce preguntas de que consta la prueba; de los datos recabados se observa que cuando más de una forma de pensamiento se evalúa en un problema, menos estudiantes lo pueden contestar correctamente. En los problema 1 y 2 se evalúa la comprensión de la conservación de una magnitud física, los problemas 3 y 4 evalúan el pensamiento proporcional, los problemas 5 a 8 evalúan la identificación y control de variables (los dos últimos incluyen probabilidad), los problema 9 y 10 analizan el pensamiento probabilístico, el 11 el pensamiento combinatorio y el 12 evalúa el pensamiento correlacional y probabilístico. La tabla 2 detalla la capacidad evaluada en cada ítem de la prueba de Lawson 
Tabla 2. Capacidades evaluadas en cada pregunta de la prueba de Lawson

\begin{tabular}{|c|l|}
\hline 1 & Conservación de masa \\
\hline 2 & Conservación del volumen desplazado \\
\hline 3 & Pensamiento de proporcionalidad \\
\hline 4 & Pensamiento avanzado de proporcionalidad \\
\hline 5 & Identificación y control de variables \\
\hline 6 & Identificación y control de variables avanzada \\
\hline 7 & Identificación y control de variables, pensamiento probabilístico \\
\hline 8 & Identificación y control de variables, pensamiento probabilístico avanzado. \\
\hline 9 & Pensamiento probabilístico \\
\hline 10 & Pensamiento probabilístico y proporcional \\
\hline 11 & Pensamiento correlacional y probabilístico \\
\hline 12 & Pensamiento combinatorio \\
\hline
\end{tabular}

Entre 1997 y 1999 se realizó un estudio aplicando el $\mathrm{FCl}$ a alumnos de la universidad de Minnesota (Henderson, 2002). En el año 2005, se publicó una investigación (Coletta, Phillips, 2005) sobre el aprendizaje de la mecánica introductoria de estudiantes de nivel universitario. El instrumento utilizado para medir el aprendizaje fue el diagnóstico $\mathrm{FCl}$ y la ganancia normalizada de Hake. El elemento nuevo que consideraron en este estudio fue la medición del razonamiento científico a través del diagnóstico de Lawson. Ellos observaron que, si bien hay una correlación débil entre el conocimiento previo del estudiante y el aprendizaje logrado, el razonamiento científico presenta una correlación mayor con el aprendizaje en cursos basados en metodologías de aprendizaje activo. Basados en esos resultados, proponen considerar el razonamiento científico en todo estudio que pretenda comparar el aprendizaje entre poblaciones diferentes con la finalidad de tener un cuadro más completo de lo que sucede (Coletta, Phillips y Steinert, 2007).

Se realizó un experimento fuera de Estados Unidos (Ates y Cataloglu, 2007) con la finalidad de conocer si hay correlaciones estadísticas entre el razonamiento científico, el conocimiento conceptual y las habilidades en la solución de problemas en un curso de mecánica introductoria. En particular se consideraron cursos con instrucción tradicional, no observándose correlación significativa entre el razonamiento científico, medido con el instrumento de Lawson, y el conocimiento conceptual, evaluado con el test $\mathrm{FCl}$ al final del curso. En México, se realizó en el Tecnológico de Monterrey, un estudio para grupos de mecánica clásica sobre la dependencia que tiene el conocimiento conceptual previo de los estudiantes y su razonamiento científico sobre el aprendizaje conceptual logrado usando una metodología de instrucción por modelación, concluyendo que muestra que el razonamiento científico juega un papel más importante que el conocimiento previo que pueda tener el estudiante al inicio del curso (Alarcón y de la Garza, 2009).

\section{LA PROPUESTA}

Este trabajo forma parte de un estudio más extenso, que pretende comparar el aprendizaje conceptual y el grado de razonamiento científico obtenido en grupos de control y grupos experimentales de mecánica clásica, en un curso con el tipo de instrucción tradicional los primeros y con la metodología de resolución explícita de problemas (Heller, Keith, 1992) los segundos. Se estudiarán las relaciones entre el estado inicial del estudiante y su aprendizaje después del curso. Se realiza un diagnóstico de la situación inicial de los alumnos que ingresan al primer curso de 
carreras relacionadas con la ciencia y tecnología: ingeniería química industrial, química industrial e ingeniería industrial. Se aplicaron dos tests a los estudiantes que ingresaron a las licenciaturas mencionadas, en la Universidad Autónoma de Yucatán, México en agosto de 2009: uno para medir su nivel de razonamiento científico y el otro para medir su conocimiento conceptual inicial de mecánica, pues a partir de enero de 2010 los cinco grupos analizados cursarían la asignatura de mecánica clásica que es parte del tronco común de sus respectivas carreras.

Los estudiantes fueron admitidos a la universidad mediante el examen EXANI-II. Esta es una prueba de selección cuyo propósito es medir las habilidades y los conocimientos básicos de los aspirantes a cursar estudios de nivel superior. Proporciona información a las instituciones sobre quiénes son los aspirantes con mayores posibilidades de éxito en los estudios de licenciatura. Es un examen de uso institucional, es decir, lo aplican instituciones (muchas universidades públicas de México) que han contratado los servicios del Centro nacional de Evaluación para la Educación Superior A.C. (CENEVAL) como parte de sus procesos de selección de aspirantes a ingresar a la educación de nivel superior. Este examen consta de 22 reactivos de razonamiento lógico matemático, 22 de matemáticas, 22 de razonamiento verbal, 22 de español y 22 de tecnologías de información y comunicación. Por otro lado, el examen diagnóstico de CENEVAL que también fue aplicado a estos estudiantes para su selección informa sobre el nivel de desempeño que guardan los sustentantes al contestar uno de ocho módulos disponibles. Para las áreas de Ciencias Naturales y exactas e Ingenierías y tecnología, el examen de diagnóstico consta de 20 reactivos de cálculo, 20 de física, 20 de química, 20 de matemáticas y 20 de inglés (CENEVAL, 2009).

Para este trabajo se tomaron en cuenta dos de los resultados de estos exámenes que proporciona el CENEVAL: el Índice de razonamiento lógico-matemático (IRLM) del examen de selección y el diagnóstico del área de Física. En este rubro se hizo una comparación descriptiva de ambos resultados pues sólo se tuvo acceso al reporte final de aplicación que proporciona en CENEVAL y no al cuerpo de los exámenes.

\section{MATERIALES Y METODOS}

El presente estudio fue realizado con 200 estudiantes de ingeniería y química industrial de primer ingreso en la Universidad Autónoma de Yucatán (UADY). Los 5 grupos utilizados en el estudio fueron distribuidos por el departamento de control escolar de la institución y, normalmente se mantienen por "paquete de asignaturas" durante los primeros semestres por lo que se tomaron como muestras intactas. En esta investigación se aplicó la prueba $\mathrm{FCl}$ casi al final del primer semestre, con la finalidad de medir el conocimiento conceptual con el que iniciarán el curso de mecánica clásica de tronco común en enero de 2010. Esta prueba puede tomar valores de 0 a 100 puntos, representando el porcentaje de aciertos del estudiante. Para establecer los conceptos que traerán los estudiantes al curso se utilizó la primera de dos aplicaciones del $\mathrm{FCl}$, a la que se llamó FClpre.

Para medir el razonamiento científico del estudiante se utilizó la prueba de Lawson, que puede tomar valores entre 0 y 100 , que representan el porcentaje de aciertos del estudiante a las 24 preguntas pareadas que tiene el instrumento. Dependiendo de lo que obtenga el estudiante en esta prueba, el estudiante puede clasificarse de acuerdo al tipo de razonamiento, que puede ser: (1) concreto, si obtiene un puntaje igual o inferior a 33.34, (2) formal, si el puntaje es mayor a 33.4 y menor a 75.00, y (3) postformal, si obtiene un puntaje superior a 75.00. Un estudiante está en el nivel más alto de la escala ordinal de razonamiento cuando consistentemente demuestra tener un razonamiento formal (Ates y Cataloglu, 2007). Con el apoyo de métodos estadísticos básicos se analizaron los resultados de estas pruebas.

Con el fin de tener una comparación de la relación entre el grado de razonamiento de los estudiantes y su desempeño en Física, se recopiló los resultados obtenidos por ellos en la prueba de ingreso del CENEVAL a la universidad. En las tablas 3 y 4 se presentan los resultados de dos de los parámetros evaluados por el CENEVAL: el Índice de razonamiento lógico-matemático (IRLM) que es uno de los cinco índices criteriales o de selección y el puntaje de diagnóstico de Física, que no formó parte del criterio de selección. 
Tabla 3. Resultados del Centro Nacional de Evaluación para la Educación Superior A.C. (CENEVAL)

\begin{tabular}{|c|c|c|c|}
\hline & \multicolumn{3}{|c|}{ Alumnos con $700 \leq \mathrm{IRLM}<1000$} \\
\hline & \multicolumn{3}{|c|}{ Diagnóstico de Física por CENEVAL } \\
\hline Grupo & Elemental (\%) & Satisfactorio (\%) & Sobresaliente (\%) \\
\hline 1 & 0.0 & 2.3 & 0.0 \\
\hline 2 & 0.0 & 0.0 & 0.0 \\
\hline 3 & 0.0 & 2.2 & 0.0 \\
\hline 4 & 0.0 & 6.7 & 0.0 \\
\hline 5 & 0.0 & 2.4 & 0.0 \\
\hline
\end{tabular}

Tabla 4. Resultados del Centro Nacional de Evaluación para la Educación Superior A.C. (CENEVAL)

\begin{tabular}{|c|c|c|c|}
\hline & \multicolumn{3}{|c|}{ Alumnos con IRLM $\geq 1000$} \\
\hline Grupo & Elemental (\%) & Satisfactorio (\%) & Sobresaliente (\%) \\
\hline 1 & 0.0 & 53.5 & 44.2 \\
\hline 2 & 8.0 & 52.0 & 40.0 \\
\hline 3 & 2.2 & 57.8 & 37.8 \\
\hline 4 & 2.2 & 57.8 & 33.3 \\
\hline 5 & 0.0 & 57.1 & 40.5 \\
\hline
\end{tabular}

Los resultados de los exámenes del CENEVAL se interpretan de la siguiente manera: La calificación numérica (índice) es una escala que se usa para reportar el resultado de los sustentantes del examen de selección y va de 700 a 1300 puntos, donde 700 representa la calificación más baja que puede obtenerse y 1300 la más alta. El puntaje que un sustentante obtiene no puede interpretarse como una calificación aprobatoria o reprobatoria, dado que el instrumento sirve para comparar el desempeño de los sustentantes con respecto de los demás que se presentaron a la aplicación. Por ejemplo, si se pide a un grupo de alumnos que brinquen lo más lejos que puedan sin impulso, la distancia que logre cada uno, sólo permite ordenarlos con base en el indicador de quién llega más lejos, pero no existen distancias máxima ni mínima a lograr. Lo que sí debe considerarse es que el examen de selección está diseñado para que la mayoría de la población que lo sustenta logre un puntaje cercano a 1000.

Para comunicar el resultado en cada una de las áreas del examen diagnóstico, se emplean niveles de desempeño que representan el nivel de conocimientos y habilidades de un sustentante: sobresaliente, satisfactorio, elemental y sin dictamen. A continuación se describe cada nivel en forma general:

Sobresaliente. El sustentante es capaz de integrar y aplicar diferentes conocimientos y habilidades para solucionar problemas novedosos o de la vida cotidiana. Maneja criterios para evaluar hechos o procedimientos y es capaz de sustentar de manera clara opiniones y juicios. Satisfactorio: El sustentante comprende los contenidos presentados. Puede expresar una misma información de formas diferentes y asociarla con otros elementos; puede extrapolar información para explicar eventos y es capaz de identificar relaciones causales, de asociación y jerárquicas.

Elemental. El sustentante reconoce las ideas principales de los textos, maneja hechos o datos como copia literal y reproduce los contenidos de un documento, haciendo uso de otras palabras.

Sin dictamen. El sustentante no contestó o todas sus preguntas fueron incorrectas. 


\section{RESULTADOS}

En la tabla 5 se presentan los resultados para las pruebas aplicadas. Estas no presentaron diferencias significativas en sus medias según lo evidenció la prueba paramétrica t de Student, con $\alpha=0.05$. Este resultado coincide con las conclusiones de Coletta y Phillips que encontraron una correlación positiva entre las puntuaciones de la prueba de Lawson y las ganancias normalizadas del FCl (Coletta, Phillips, 2005), aunque en este caso sólo se comparan los puntajes de la primera aplicación del FCl.

Tabla 5. Resultados por grupo de la aplicación de la prueba del Lawson y del FCl

\begin{tabular}{|c|c|c|c|c|c|}
\hline \multicolumn{2}{|c|}{} & \multicolumn{2}{c|}{ Conocimiento conceptual } & \multicolumn{2}{c|}{ Razonamiento científico } \\
\hline Grupo & $\mathrm{n}$ & Media FCIpre & Desv. Est. & Media Lawson & Desv. Est. \\
\hline 1 & 43 & 29.69 & 7.26 & 51.83 & 13.85 \\
\hline 2 & 25 & 29.73 & 7.19 & 51.05 & 13.87 \\
\hline 3 & 40 & 28.00 & 5.87 & 41.11 & 14.03 \\
\hline 4 & 40 & 28.81 & 7.32 & 49.16 & 15.24 \\
\hline 5 & 42 & 26.90 & 5.62 & 42.26 & 16.81 \\
\hline
\end{tabular}

La figura 1 muestra el porcentaje de alumnos en cada nivel de razonamiento, medido por la prueba de Lawson. Fueron clasificados por grupo los pensadores concretos, los formales y los postformales tomando en cuenta el número de respuestas correctas en la prueba.

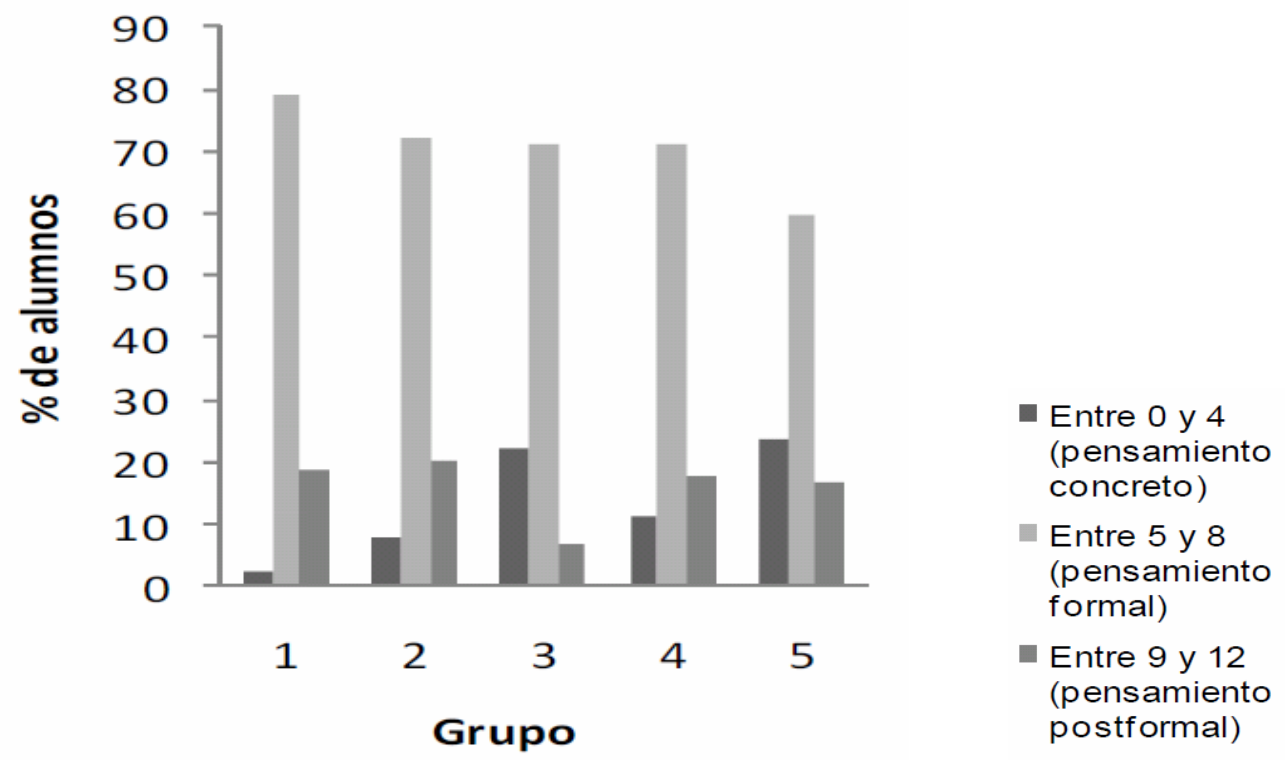

Fig.1: Porcentaje de alumnos en cada nivel de razonamiento para cada grupo

La figura 2 muestra el porcentaje de aciertos por grupo y por concepto de acuerdo a la prueba $\mathrm{FCl}$. Se puede observar que, en el rubro de cinemática más del $40 \%$ de los estudiantes presenta errores conceptuales. Como ejemplo, los estudiantes tuvieron problemas en conceptos tan básicos como el de aceleración. Los resultados son peores en el rubro de la Segunda Ley de Newton, en más del $70 \%$ de los estudiantes presenta errores conceptuales. Un ejemplo es la incidencia de la idea pre-newtoniana de que se necesita una fuerza para mantener un cuerpo en movimiento rectilíneo uniforme. Dos preguntas tuvieron resultados muy bajos. La primera tiene que ver con la suma de velocidades como cantidades vectoriales. Para la mayoría de los alumnos, las velocidades se suman aritméticamente o la velocidad resultante de un objeto después de un golpe no depende de la velocidad que tenía el objeto antes del golpe. La segunda pregunta tiene que ver con el efecto de una fuerza sobre un objeto. La mayoría de las respuestas están relacionadas con una idea muy frecuente en los alumnos: a fuerza doble, velocidad doble. 


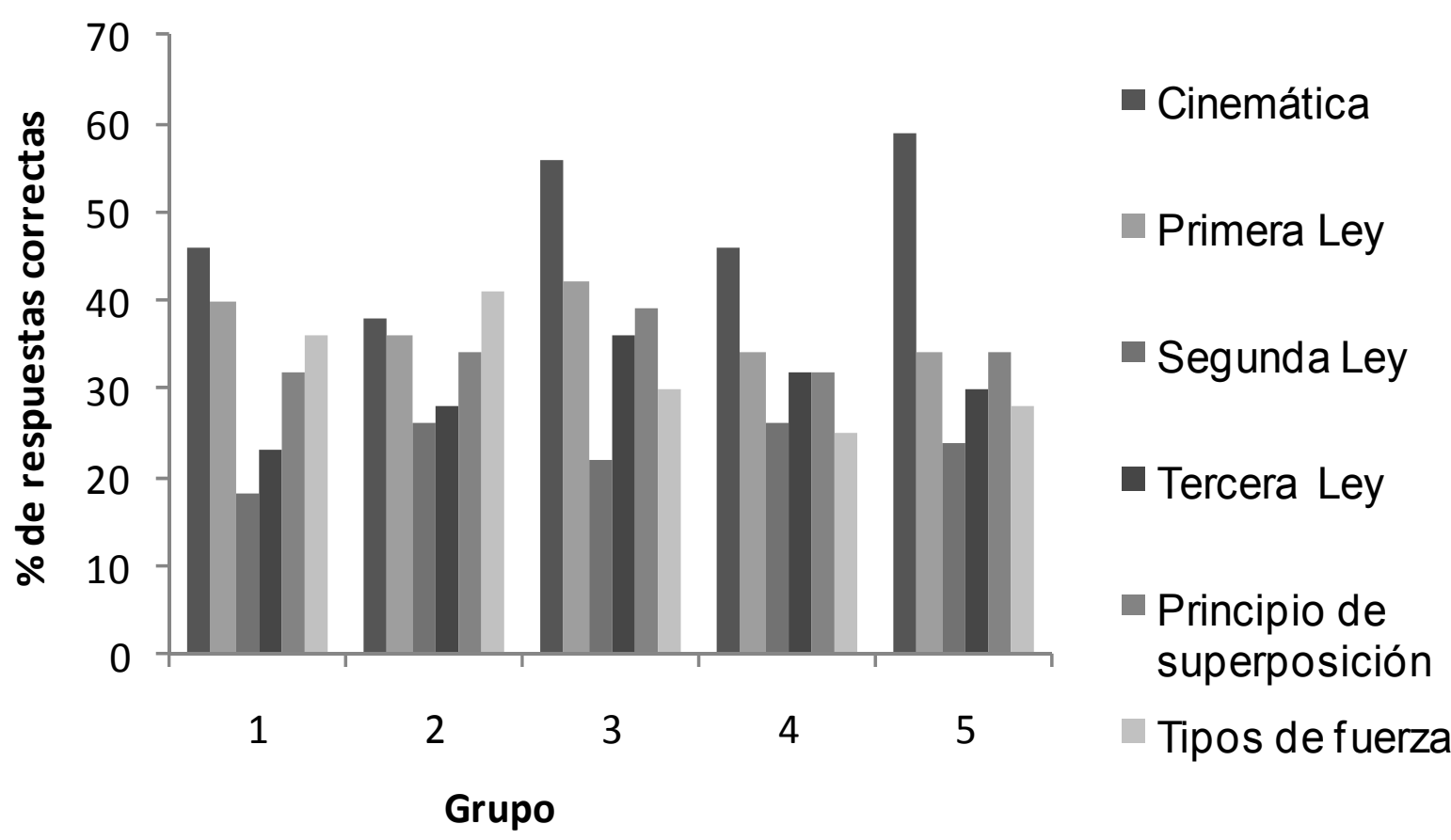

Fig.2: Porcentaje de aciertos por concepto de la prueba FCl para cada grupo

De los estudios realizados, se observa que de los ingresantes a las licenciaturas en ingeniería y química industrial de los cinco grupos estudiados un bajísimo porcentaje razona como pensadores formales antes de abordar el curso introductorio de mecánica. Destaca el bajo porcentaje de estudiantes post-formales (capaces de probar hipótesis causales observables y noobservables) y el porcentaje que permanece en la etapa concreta (necesidad de operar directamente con los objetos). El rango entre $36 \%$ y $62 \%$ de alumnos en nivel concreto indica la situación real de los alumnos que concluyen el bachillerato. Esto pudiera estar relacionado con un entendimiento frágil de los conceptos físicos y matemáticos involucrados, los cuales han sido adquiridos en su mayoría vía definiciones y formalismos matemáticos (Ruiz, Fuchs y Raggi, 2007).

De acuerdo a la prueba de Lawson, los estudiantes tienen dificultades para resolver problemas que involucran el pensamiento proporcional avanzado (pregunta $4 ; 23 \%$ de aciertos) y el pensamiento probabilístico avanzado (pregunta 10; $21 \%$ de aciertos). Sin embargo las tareas que más dificultades causaron a los estudiantes fueron las relacionadas con la identificación y control de variables combinada con probabilidad (preguntas 7 y 8 ; alrededor de $15 \%$ de aciertos), el pensamiento combinatorio (pregunta $11 ; 8 \%$ de aciertos) y el pensamiento correlacional combinado con probabilidad (pregunta 12; 3\% aciertos). Si los estudiantes que han ingresado a estas licenciaturas han tenido históricamente estas dificultades iniciales en cuanto a nivel de razonamiento, no es de sorprender que los estudiantes hayan tenido serias dificultades para enfrentar los cursos de física de los primeros semestres. Es conveniente que en los cursos de física se diseñen e implementen actividades que permitan que estas formas de razonamiento se fortalezcan.

De la aplicación de la prueba de Lawson también se deduce que los estudiantes tienen dificultades para comprender un texto, que las partes de un texto que no llegan a comprender, las ignoran y esto repercute en la calificación que logran obtener en la prueba. Todo parece indicar que el nivel de razonamiento del estudiante está relacionado con la comprensión de textos y con una base sólida en cuanto a los conceptos de física, aunque para encontrar la forma en que estas tres partes están relacionadas, se requeriría un estudio detallado. 
Con respecto a los resultados de la aplicación del $\mathrm{FCl}$, en la tabla 5 se observa que la media de todos los grupos examinados no supera los 30 puntos y que, analizando los resultados por rubro (figura 2), los mejores resultados en los cinco grupos se presentaron en los conceptos de cinemática aunque los porcentajes de resultados correctos no rebasaron el $60 \%$. Por otro lado los peores resultados en todos los casos estuvieron relacionados con la segunda ley de Newton con un porcentaje máximo de aciertos de $25 \%$.

En las tablas 3 y 4 se observa, de manera general con respecto a los resultados de CENEVAL que los estudiantes con un IRLM mayor o igual a 1000 puntos presentan consistentemente en los cinco grupos un mayor porcentaje de alumnos clasificados como "sobresaliente" en el diagnóstico de Física. De forma análoga, los alumnos con un IRLM menor a 1000 puntos presentaron en todos los casos un porcentaje mayor de alumnos clasificados como en nivel "satisfactorio" del diagnóstico de Física. No obstante estos resultados en el salón de clase los profesores han manifestado que los estudiantes presentan carencias significativas en sus bases teóricas, con lo que se cuestiona los resultados del CENEVAL en la prueba de física. Una prueba estadística de correlación con un nivel de significancia de $\alpha=0.05$ demostró dependencia lineal entre los resultados de la prueba de Lawson, de FCI y el IRLM del CENEVAL. No pudo realizarse la prueba respecto a los resultados de Física debido a que esa sección del CENEVAL no es de acceso público a sus resultados numéricos.

\section{CONCLUSIONES}

Se obtuvo un porcentaje significativo de estudiantes con un nivel de razonamiento científico bajo, lo que los clasifica como pensadores concretos y formales, requiriéndose, a nivel universitario, un nivel de razonamiento postformal. La aplicación del $\mathrm{FCl}$ mostró porcentajes de acierto bajos en todos los rubros, principalmente los relacionados con Segunda Ley de Newton. Se encontró relación estadística entre los resultados obtenidos por la prueba de Lawson y la del FCl.

De los resultados del examen de selección EXANI-II aplicado por el Centro Nacional de Evaluación para la Educación Superior (CENEVAL, México) se encontró correlación entre las puntuaciones del Índice de Razonamiento Lógico Matemático (IRLM) y los resultados de la prueba de Lawson y la del FCI. Se cuestiona la eficacia para diagnosticar los conocimientos y habilidades de física mediante la prueba del CENEVAL.

Los resultados por grupo y los resultados individuales de los estudiantes en todas las mediciones analizadas servirán como datos iniciales de comparación con los resultados de aplicar a estos grupos una estrategia de resolución explícita de problemas en el curso de mecánica que iniciaron en enero de 2010. Esto con el fin de determinar si se puede hablar de una mejora en los niveles de razonamiento científico y en la comprensión de los conceptos en mecánica Clásica.

\section{REFERENCIAS}

Alarcón, H. y De la Garza, J. (2009). Influencia del razonamiento científico en el aprendizaje de conceptos en Física Universitaria: Comparación entre instrucción tradicional e instrucción por modelación. Memorias X congreso nacional de investigación educativa COMIE (2009).

http://www.comie.org.mx/congreso/memoria/v10/pdf/area_tematica_05/ponencias/1189-F.pdf.

Acceso: 1 de diciembre (2009)

Ates, S. y Cataloglu, E. The effects of students' reasoning abilities on conceptual understandings and problem-solving skills in introductory mechanics, European Journal of Physics, 28(6), 11611171. (2007).

CENEVAL (Centro Nacional de Evaluación para la educación Superior. A.C.) http://www.ceneval.edu.mx/ceneval-web/content.do?page=0 Acceso: 1 de noviembre (2009).

Coletta, V. P. y Phillips, J. A. Interpreting FCl scores: Normalized gain, preinstruction scores, and scientific reasoning ability, American Journal of Physics, 73(12), 1172-1182. (2005). 
Coletta, V. P.; Phillips, J. A. y Steinert, J. J. Why you should measure your students'reasoning ability, The Physics Teacher, 45(4), 235-238. (2007).

Coletta, V. P.; Phillips, J. A. y Steinert, J. J. Interpreting force concept inventory scores: Normalized gain and SAT scores, Physical review special topics - physics education research 3 , 010106 (2007).

Concari, S. El modelado y la resolución de problemas: ejes para la enseñanza de la Física para ingenieros Contextos de educación 5 .Universidad de Río Cuarto, Argentina. (2002). http://www.unrc.edu.ar/publicar/cde/05/Concari.htm. Acceso: 15 de marzo (2009).

Diff, K. y Tache, N. From FCI To CSEM To Lawson Test: A Report On Data Collected At A Community College. AIP Conf. Proc. 951, 85 (2007).

Hake, R. R. Interactive-engagement versus traditional methods: A six-thousand student survey of mechanics test data for introductory physics courses, American Journal of Physics, 66(1), 64-74. (1998).

Heller, P., Keith, R. y Anderson, S. Teaching problem solving through cooperative grouping. Part 1: Group versus individual problem solving. Am. J. Phys. 60 (7), 627-727 (1992).

Henderson, H. Common concerns about the Force Concept Inventory, the physic teacher, 40, 542547. (2002).

Hestenes, D.; Wells, M. y Swackhamer, G. Force concept inventory, The Physics Teacher, 30(3), 141-158. (1992).

Leonard, W., Gerace J. y Dufresne, J. Resolución de problemas basada en el análisis. Hacer del análisis y del razonamiento el foco de la enseñanza de la física. Enseñanza de las ciencias: revista de investigación y experiencias didácticas, 20(3), 387-400 (2002).

Pozo, J. y otros cinco autores, Nuevas formas de pensar la enseñanza y el aprendizaje, $1^{a}$ edición, Graó, España, 50-55. (2006).

Renner, J. W. y Lawson, A. E. Piagetian theory and instruction in physics, The Physics Teacher, 11(3), 165-169. (1973).

Ruiz, H., Fuchs, L. y Raggi, G. El desarrollo del pensamiento científico de los ingresantes a las licenciaturas de FCFM-BUAP y su adaptación a los estudios. III Encuentro Participación de la mujer en la ciencia. CIATEC, (2007). 